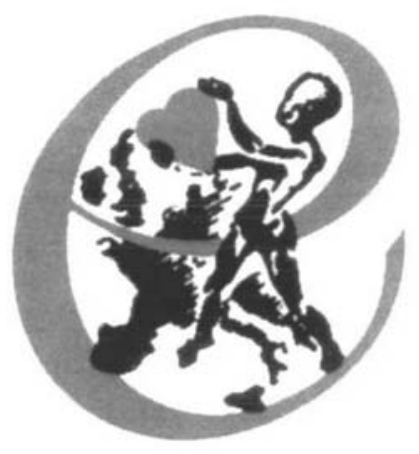

\title{
AEPC Newsletter
}

\section{What is happening with the European Paediatric Cardiac Code?}

\author{
Franco P. Stocker (Chairman, Coding Committee, Association for European \\ Paediatric Cardiology)
}

$\mathrm{F}$ OR QUITE SOME TIME, PAEDIATRIC CARDIOLOGISTS and paediatric cardiac surgeons in Europe have felt the need for common diagnostic and therapeutic codes for categorisation of infants and children with congenital and acquired heart disease. On these grounds, the Association for European Paediatric Cardiology formed a coding committee, which met for the first time during the annual meeting held in Stockholm in 1997. The members of this committee, Robert $H$. Anderson, Otto Daniëls, Martin Elliott, Rodney Franklin, Marc H. Gewillig, Otto N. Krogmann, Renzo Ghisla, Franco P. Stocker, and Herbert E. Ulmer, established first the principles which would underscore the required method of coding. In essence, it was necessary to provide a detailed and long coding list, requested by many of our members, but at the same time to satisfy the equal need for a much shorter list, albeit still complete, which was compatible with, and hence based upon, the detailed long list. This meant that, in the short list, detailed terms were needed on the one hand which were important or frequently used, but on the other hand these many terms had to be put in the context of "collecting items". In this way, it was possible for the short coding list, which then contained the collecting items, also to be complete. As an extension of this principle, if the short list is based exclusively on the detailed long list, it will then obviously be possible, according to individual needs, to combine the two lists if considered either necessary or appropriate.

As their first step, therefore, the coding committee proposed the adoption of these principles to the annual assembly of the Association held in Dublin in 1998. Their additional proposal was that

Correspondence ro: Franco P. Stocker, Chairman, Coding Committee, Association for European Paediatric Cardiology, Division of Paediatric Cardiology, University Children's Hospital, CH-3010 Bern, Switzerland. the existing extensive codes derived initially from the Brompton Hospital Code, refined by a group of Dutch workers, and subsequently additionally modified by Martin Elliott and Rodney Franklin, should form the basis of the detailed long list. Both propositions were accepted, and the coding committee was next asked to prepare a suitable short list for presentation to the forthcoming meeting in Sofia. They were also asked to update and correct the existing long list, so that the two together could form the future European Paediatric Cardiac Code. This task has been now been achieved, partly by written discussions between members of the committee, but mainly during a meeting held in London in November of 1998, The Coding Committee is now able to provide an updated and detailed long list, comprising around four to five thousand items which provide comprehensive codes not only for diagnosis but also for therapeutic procedures. This is then interchangeable with a complete short list, again suitable for both diagnostic and therapeutic needs, which contains no more than five to six hundred items. This short list has now been distributed, along with a questionnaire inviting comments and criticisms, to all those centres in Europe which initially signalled their interest in a common European code. Both lists will be presented for ratification by the annual assembly in Sofia.

Many centres are now also obliged, by either their hospital or political administrations, to use the ninth and tenth revisions of the International Classification of Disease for the purposes of coding. Because of this, the coding committee has made it possible to link the short and long versions of the European Paediatric Cardiac Code with both these revisions of the International Code. A similar match will be provided for the eleventh revision when it becomes available. This will facilitate greatly the process of coding individual patients, since if the patient is coded using the European 
system, an automatic match will be provided for the recent revisions of the International Classification. It is axiomatic, therefore, that use of the proposed European code will facilitate research and assurance of quality not only for paediatric cardiologists, but also for paediatric cardiac surgeons. Because of this, we have taken care to have the European paediatric cardiac surgeons properly represented in the coding committee. It is now our intention also to present our final propositions for ratification by the European paediatric cardiac surgeons.
As soon as the long and short versions of the Code are accepted by the Association for European Paediatric Cardiology, and by the European paediatric cardiac surgeons, both lists will be published in Cardiology in the Young, along with a commentary on their use. Any member of the association wishing to express personal views on the process of coding, or wanting to make further individual contributions, is urged to attend the forthcoming meeting in Sofia, or alternatively to write directly to myself as Chairman of the coding committee. 